\title{
TTR
}

Traduction, terminologie, rédaction

\section{Promouvoir le transculturel comme « typiquement allemand »? Retour sur cinquante ans de littérature de la migration et sur sa promotion}

\section{Sarah Neelsen}

Volume 31, numéro 2, 2e semestre 2018

Minorité, migration et rencontres interculturelles : du binarisme à la complexité

Minority and Migrant Intercultural Encounters: From Binarisms to Complexity

URI : https://id.erudit.org/iderudit/1065574ar

DOI : https://doi.org/10.7202/1065574ar

Aller au sommaire du numéro

Éditeur(s)

Association canadienne de traductologie

ISSN

0835-8443 (imprimé)

1708-2188 (numérique)

Découvrir la revue

Citer cet article

Neelsen, S. (2018). Promouvoir le transculturel comme « typiquement allemand " ? Retour sur cinquante ans de littérature de la migration et sur sa promotion. TTR, 31(2), 195-217. https://doi.org/10.7202/1065574ar

\section{Résumé de l'article}

Cet article considère la question du transculturalisme dans la littérature contemporaine de langue allemande, dite " littérature de la migration ». Bien que l'importance de cette littérature se soit accrue dans tous les pays de l'aire germanophone, l'accent est mis ici sur l'Allemagne. L'article donne un aperçu des différentes vagues de migrants qui sont arrivées en Allemagne après la Seconde Guerre mondiale : les populations d'Europe centrale et orientale, expulsées de leur pays de résidence entre 1944 et 1950, les Gastarbeiter venus sur des contrats de travail temporaires entre 1950 et 1970, les réfugiés politiques durant la guerre froide et les migrants économiques après l'effondrement du bloc soviétique, ainsi que quelques représentants de la mobilité individuelle et librement choisie dans un passé plus récent. Chacune de ces vagues a produit des écrivains qui ont rédigé tout ou partie de leur oeuvre en allemand, le plus souvent sous l'influence d'autres langues avec lesquelles ils avaient été en contact. Ces textes témoignent d'une situation entre les langues vécue très différemment selon les auteurs. Sont évoqués Rafik Shami et Feridun Zaimoğlu, mais aussi Herta Müller, Terézia Mora, Sharon Dodua Otoo et Yoko Tawada. Si cette littérature est largement connue et étudiée dans les pays de langue allemande et dans les départements d'études germaniques des universités, le présent article prend le parti d'interroger la façon dont elle est promue par la politique culturelle, le marché du livre et les historiens de la littérature.
Ce document est protégé par la loi sur le droit d'auteur. L’utilisation des services d’Érudit (y compris la reproduction) est assujettie à sa politique d'utilisation que vous pouvez consulter en ligne.

https://apropos.erudit.org/fr/usagers/politique-dutilisation/ 


\section{Promouvoir le transculturel comme "typiquement allemand»? Retour sur cinquante ans de littérature de la migration et sur sa promotion}

\section{Sarah Neelsen}

Université Sorbonne Nouvelle - Paris 3

\section{Résumé}

Cet article considère la question du transculturalisme dans la littérature contemporaine de langue allemande, dite «littérature de la migration». Bien que l'importance de cette littérature se soit accrue dans tous les pays de l'aire germanophone, l'accent est mis ici sur l'Allemagne. L'article donne un aperçu des différentes vagues de migrants qui sont arrivées en Allemagne après la Seconde Guerre mondiale: les populations d'Europe centrale et orientale, expulsées de leur pays de résidence entre 1944 et 1950, les Gastarbeiter venus sur des contrats de travail temporaires entre 1950 et 1970, les réfugiés politiques durant la guerre froide et les migrants économiques après l'effondrement du bloc soviétique, ainsi que quelques représentants de la mobilité individuelle et librement choisie dans un passé plus récent. Chacune de ces vagues a produit des écrivains qui ont rédigé tout ou partie de leur œuvre en allemand, le plus souvent sous l'influence d'autres langues avec lesquelles ils avaient été en contact. Ces textes témoignent d'une situation entre les langues vécue très différemment selon les auteurs. Sont évoqués Rafik Shami et Feridun Zaimoğlu, mais aussi Herta Müller, Terézia Mora, Sharon Dodua Otoo et Yoko Tawada. Si cette littérature est largement connue et étudiée dans les pays de langue allemande et dans les départements d'études germaniques des universités, le présent article prend le parti d'interroger la façon dont elle est promue par la politique culturelle, le marché du livre et les historiens de la littérature.

Mots-clés : littérature allemande, littérature de la migration, interculturalité, politique culturelle de l'Allemagne, langue allemande dans le monde

\section{Abstract}

This article considers the question of transculturalism in contemporary German-language literature called "literature of migration." Although its importance has increased in all German-speaking countries, the focus 
here is on Germany. The article gives an overview of the different groups of migrants who arrived in Germany after the Second World War: Central and Eastern European populations expelled from their countries of residence between 1944 and 1950, Gastarbeiters who came on temporary employment contracts between 1950 and 1970, political refugees during the Cold War and economic migrants after the collapse of the Soviet bloc, as well as some representatives of individual and freely chosen mobility in the more recent past. Each of these waves produced writers who wrote all or part of their work in German, often influenced by other languages with which they had been in contact. These texts testify to a situation between languages experienced very differently according to the authors. Rafik Shami and Feridun Zaimoğlu, but also Herta Müller, Terézia Mora, Sharon Dodua Otoo, and Yoko Tawada serve as examples. While this literature is widely known and studied today in German-speaking countries and in post-secondary departments of German Studies, the present article questions the way in which it is promoted by German cultural policy, the book market, and historians of literature.

Keywords: German literature, literature of migration, interculturality, German cultural policy, German language in the world

\section{Introduction}

Depuis 2015, l'attention des médias internationaux est focalisée sur l'arrivée de plus d'un million de réfugiés en sol allemand et sur la façon dont louverture des frontières, décidée par la chancelière Angela Merkel, modifiera le visage de l'Allemagne et de l'Europe. Pourtant, un tel changement est déjà à l'œuvre depuis longtemps. La récente "crise migratoire» a servi surtout de révélateur d'une diversité démographique et culturelle alors que l'image de l'Allemagne restait pour beaucoup empreinte des clichés hérités des discours nationalistes des $\mathrm{XIX}^{\mathrm{e}}$ et $\mathrm{XX}^{\mathrm{e}}$ siècles. La littérature allemande est à ce titre un exemple paradigmatique de la manière dont la culture allemande a longtemps été perçue : "hermétique, compliquée et exigeante» (Reimann, 2017, p. 116) du point de vue stylistique, et thématisant forcément l'histoire des deux dictatures qu'a connues le pays au siècle dernier. Un entretien de 2017 entre les écrivains Christoph Ransmayr et Olivier Rolin ${ }^{1}$, ténors de la littérature con-

1. Entretien du 9 septembre 2017 au Palais du Gouvernement de Nancy, dans le cadre du salon littéraire "Le Livre sur la Place». L'entretien était mené par le directeur de l'Institut Goethe de la ville, Nicolas Ehler, qui a volontairement mis en avant, durant cet échange, les relations entre les cultures et les littératures de langues allemande et française. Le fait que Ransmayr soit autrichien n'a joué qu'un rôle mineur, symptôme de la perception de la littérature de langue allemande à l'étranger, où les différents espaces de l'aire germanophone sont rarement distingués. 
temporaine dans leur pays respectif (l'Autriche pour Ransmayr, la France pour Rolin), rend bien compte de la situation : interrogés sur leurs influences, le premier cita sans hésiter Balzac, Flaubert et Proust, mais aussi les contemporains Patrick Deville et Mathias Enard ${ }^{2}$; le second ne sut convoquer qu'un lointain souvenir de la nouvelle La métamorphose (1915) de Franz Kafka et son intention de lire un jour le monumental roman de Thomas Mann, La montagne magique (1928). Les quelques œuvres qui ont suscité l'attention du public ces dernières années - souvent dans l'ignorance qu'il s'agissait d'œuvres allemandes et certainement sans distinguer entre l'Allemagne, la Suisse et l'Autriche - ont été des adaptations cinématographiques (La pianiste, Le parfum ou encore Le liseur) ${ }^{3}$. C'est cet arrière-plan qui permet de mesurer la petite révolution qui s'est produite récemment : l'une des distinctions littéraires les plus importantes des pays de langue allemande, le Prix Ingeborg Bachmann, a été décernée en 2016 à Sharon Dodua Otoo (née en 1972), écrivaine britannique d'origine ghanéenne, pour Herr Gröttrup setzt sich bin [Monsieur Gröttrup s'assoit], comme si les jurés avaient voulu clairement signifier que l'image qu'on s'est longtemps faite de la littérature de langue allemande et de ses auteurs devait être révisée en profondeur

La $40^{\mathrm{e}}$ lauréate du Prix Ingeborg Bachmann illustre deux phénomènes majeurs. Premièrement, l'existence d'une politique volontariste $^{4}$ au sein de l'aire germanophone pour accueillir de nouvelles voix en littérature. Deuxièmement, une recrudescence d'auteurs allophones prêts à faire de l'allemand leur langue d'écriture, alors même qu'ils ont ou qu'ils pourraient avoir accès au marché anglophone dont

2. Du premier, Patrick Deville, on peut citer ici Peste et choléra (2012), récompensé du Prix Femina, tandis que Mathias Enard est notamment l'auteur de Zone (2008) et Boussole (2015), pour lequel il a reçu le Prix Goncourt.

3. Le roman d'Elfriede Jelinek, Die Klavierspielerin (La pianiste), publié en 1983, a été adapté au cinéma par Michael Haneke en 2001 avec Isabelle Huppert, Annie Girardot et Benoît Magimel dans les rôles principaux. Il a été récompensé de trois prix au Festival de Cannes. Das Parfum (1985) (Le parfum) de Patrick Süskind, roman à grand succès, a fait l'objet d'une adaptation en 2006 par Tom Tykwer (Prix du cinéma européen 2007). Enfin, Der Vorleser (Le liseur) de Bernhard Schlink, paru en 1995, best-seller traduit en une quarantaine de langues, a inspiré en 2008 le film de Stephen Daldry The Reader. L'actrice Kate Winslet a reçu un Oscar, un Golden Globe et un BAFTA pour le rôle de Hannah.

4. Dodua Otoo ignorait l'existence même de ce prix avant d'être invitée à soumettre sa candidature. D'où d'ailleurs ce commentaire humoristique dans les colonnes de la Frankfurter Allgemeine Zeitung : "C'est la preuve que des auteurs britanniques ignorent encore qu'ils seraient excellents en allemand»(Kaube, 2016; ma trad.). 
on sait l'importance quantitative ${ }^{5}$. L'objet de cet article sera de mettre en perspective ce phénomène en apparence nouveau, à travers une approche historique des différentes vagues migratoires qu'a connues l'Allemagne après la Seconde Guerre mondiale. On interrogera pour chaque vague la façon dont les auteurs se sont approprié la langue allemande et ont problématisé les rapports entre leur(s) langue(s) et culture(s) première(s) et celle(s) rencontrée(s) dans le pays d'accueil. Lobjectif est de montrer comment l'Allemagne s'est mise à valoriser la réalité plurilingue et transculturelle de sa société pour renouveler son identité après la réunification. Un renouvellement qui ne va pas sans difficulté, puisqu'il aboutit, on le verra, à vouloir faire du transculturel le «typiquement allemand».

\section{Les travailleurs migrants des années 1950 à 1970 : des écrivains de l'ombre}

On estime qu'un cinquième de la population allemande actuelle est issu de l'immigration, soit 18,6 millions de personnes, dont la moitié possède la nationalité allemande (Destatis, 2017). Le terme allemand retenu par l'Office fédéral des statistiques (Statistisches Bundesamt, encore appelé Destatis), à savoir Menschen mit Migrationshintergrund, inclut à la fois les personnes ayant une expérience personnelle de la migration et leurs descendants, si au moins un des parents est migrant ou naturalisé. Parmi ces personnes, 6,42 millions proviennent de pays avec lesquels l'Allemagne a conclu des accords migratoires à partir de 1955 (Italie) et au cours des années 1960 (Espagne, Grèce, Turquie, Maroc, Portugal, Tunisie, Yougoslavie), jusqu'en $1973^{6}$. L'arrivée de ces populations ouvrières est considérée - à tort, comme on le verra plus loin - comme le début de l'histoire migratoire de l'Allemagne contemporaine (Destatis, 2013), et la Gastarbeiterliteratur (littérature des travailleurs migrants ou, littéra-

5. Si l'on considère que le Prix Ingeborg Bachmann est décerné en Autriche, on pourrait ajouter un troisième phénomène à cette liste, à savoir que l'impulsion donnée par l'Allemagne en matière de promotion de l'interculturalité est clairement relayée dans le reste de l'aire germanophone.

6. Cette main-d'œuvre étrangère, dont le contrat ne dépassait pas en règle générale les trois ans, était appelée Gastarbeiter en Allemagne de l'Ouest et Vertragsarbeiter en Allemagne de l'Est (originaires de pays socialistes: Vietnam, Mozambique, Pologne, Angola, Cuba). On estime que 14 millions de personnes sont venues en Allemagne dans le cadre de ces conventions de travail bilatérales et qu'entre deux et trois millions sont restées, bénéficiant plus tard du regroupement familial (Wehler, 2008, p. 41; Oltmer 2012, p. 11). 
lement, des travailleurs invités) est généralement placée elle aussi aux origines de la littérature de la migration ${ }^{7}$ dans les histoires littéraires (Herrmann et Horstkotte, 2016, p. 124-125).

Quantitativement, la production littéraire de cette époque n'a pas été très importante, et peu d'auteurs ont réussi à s'imposer dans le temps. On peut citer les Italiens Carmine Gino Chiellino (né en 1946) et Franco Biondi (né en 1947), auteurs de recueils poétiques parus entre la fin des années 1970 et le début des années 1980, et qui sont passés ensuite au genre romanesque. Généralement, ce sont surtout les auteurs turcs qu'on retient, Emine Sevgi Özdamar avec Das Leben ist ein Karawanserei (1992) et Die Brücke vom Goldenen Horn (1998), et Feridun Zaimoğlu, auteur du polémique Kanak Sprak (1995). La première (née en 1946) a fait partie des femmes, beaucoup moins nombreuses, à être venues pour travailler en usine en Allemagne de 1965 à 1967. Elle y est retournée en 1971 pour entamer une carrière d'actrice entre Berlin et la France, et elle écrit aujourd'hui aussi des pièces de théâtre en turc. Le second (né en 1965) est arrivé en Allemagne à l'âge d'un an, comme fils de travailleurs migrants. Le titre de son premier livre fait référence au terme d'abord péjoratif Kanake employé pour désigner les immigrés turcs, mais que certains descendants de la deuxième et troisième génération se sont approprié et revendiquent. Arrivé à la même époque, mais comme étudiant, Suheil Fadel (né en 1946), d'origine syrienne, figure parmi les auteurs les plus vendus, avec plus d'une cinquantaine de romans traduits en trente langues et publiés sous le pseudonyme Rafik Schami. Alors qu'il compte parmi les pères de la Gastarbeiterliteratur - en tant que co-fondateur en 1980 de Südwind, l'un de ses principaux organes de publication - on considère qu'il est aussi celui «qui a réussi à s'en détacher» (Wikipédia, n. d.; ma trad.).

Le fait que cette première littérature de la migration ne compte qu'un nombre relativement faible d'auteurs (dont je n'ai ici retenu que quelques exemples) tient à deux facteurs, intimement corrélés. D'une part, l'absence délibérée d'une politique d'intégration des travailleurs migrants à cette époque, l'État ayant toujours considéré leur séjour comme temporaire. Par conséquent, peu d'entre eux ont vraiment

7. On utilise en allemand les termes Migrantenliteratur, Literatur der Migration, interkulturelle Literatur ou encore Literatur der Mehrsprachigkeit. Si je retiens ici celui de littérature de la migration, c'est parce que je mets l'accent sur l'histoire migratoire du pays. On trouvera un éclairage terminologique notamment dans Keiner (1999). 
appris la langue allemande, encadrés la journée par une interprète bilingue employée par l'usine, et hébergés la nuit dans des centres d'accueil non mixtes, souvent à l'écart du reste de la population (cf. Özdamar, 1998, chapitre 1). D'autre part, les quelques textes qui ont vu le jour dans les années 1980 et 1990 étaient fréquemment refusés par les grandes maisons d'édition (Tantow, 1984) et n'avaient de chance de publication que dans la collection Südwind ou chez l'éditeur réputé «de gauche», Neuer Malik Verlag. Il s'agissait le plus souvent de récits de travail ou de poèmes revendicatifs ancrés dans un contexte historique et politique auquel ils ont eu du mal à survivre. Zaimoğlu estime que ce qui a été encouragé chez les travailleurs migrants, c'est «une littérature larmoyante, conciliante et subventionnée [...] qui répand[ait] depuis les années 1970 le mythe du pauvre Turc au grand cœur, Ali» (1995, p. 11). La valeur documentaire qu'on a pu lui prêter un temps se voit aujourd'hui reléguée au second plan, au profit d'une «expérimentation poétique » avec les langues. Ainsi, Esther Kilchmann retrace dans ses recherches sur la littérature migratoire une évolution qui commence par des récits principalement autobiographiques et aboutit à des textes plus expérimentaux dans lesquels le contact entre les langues est source de l'invention poétique (2017, p. 178).

Pourtant, il n'est pas tout à fait exact de privilégier la source autobiographique de la Gastarbeiterliteratur, à moins de l'entendre exclusivement comme littérature produite par les travailleurs migrants. Ceux qui, comme Rafik Schami, ont pu revendiquer cette étiquette n'étaient pas eux-mêmes ouvriers, et s'ils se disaient tels, c'était dans un geste politique visant à dénoncer le sort des immigrés et leur séparation systématique d'avec les Allemands (Tantow, 1984). Cette séparation semble se poursuivre dans l'histoire littéraire, où il est rare qu'on prenne en considération les artistes allemands s'exprimant sur la question. Par exemple, l'auteur de littérature jeunesse HansGeorg Noack (1926-2005) racontait en 1973 dans Benvenuto heißt willkommen la trajectoire d'un groupe d'immigrés italiens partis d'un petit village et venus travailler à la chaîne, bientôt rejoints par leurs familles. L'auteur abordait de front la question de la construction défaillante du masculin à travers l'expérience de la migration : le père de Benvenuto, bientôt licencié et donc coupé de sa communauté, se retrouve à porter des cagettes pour un marchand de fruits et légumes, tandis que son fils, d'abord objet d'une curiosité sympathique à l'école, rate le baccalauréat et devient vendeur de glaces. Le réalisateur 
Rainer Werner Fassbinder (1945-1982) avait aussi abordé la question des travailleurs migrants sous un angle similaire, d'abord dans son film Katzelmacher (1969), dont le personnage central (interprété par Fassbinder lui-même) était un travailleur grec, puis dans son célèbre Angst essen Seele auf (1974; titre français: Tout le monde s'appelle Ali), dans lequel son compagnon E1 Hedi ben Salem, de père marocain et de mère tunisienne, incarnait le rôle-titre. D'abord ami puis époux de la femme de ménage Emmi, veuve allemande bien plus âgée que lui, Ali est filmé avant tout comme un corps, presque une ombre, quasi muet, objet de tous les fantasmes, nourris tant par la peur que par le désir.

Beaucoup de ces auteurs de la Gastarbeiterliteratur ont été récompensés entre 1985 et 2017 par le Prix Chamisso de la fondation privée Robert Bosch, destiné à des auteurs allophones écrivant en allemand. L'étiquette "Chamisso-Literatur», parfois utilisée de manière dépréciative, a pu créer un effet de groupe qui dissimule mal l'hétérogénéité des œuvres et des parcours. Natif de Champagne, Adelbert von Chamisso (1781-1838) était de langue maternelle française, mais est allé à l'école à Berlin après que ses parents eurent fui devant les Révolutionnaires. Européen avant l'heure, il aurait montré l'exemple d'une existence entre les langues, entre les cultures allemande et française, avec la publication en allemand de Peter Schlemibls wundersame Geschichte en 1813. Or, c'est peut-être moins l'homme Chamisso que sa créature, Peter Schlemihl, qui est la véritable figure tutélaire des premiers lauréats du Prix, ceux qui appartenaient à cette première vague migratoire. Arrivée par bateau en terre étrangère, Schlemihl rencontre en chemin un homme "gris et sec» qui lui demande son ombre en échange d'une grande quantité d'or. Privé de son ombre, Schlemihl se retrouve à vivre en marge de la société, à ne sortir que les nuits sans lune, rejeté par le plus grand nombre. Refusant un second marché, Schlemihl entame une longue errance qui fera de lui un citoyen du monde, capable d'aller faire sécher au soleil de Libye ses pieds qu'il vient de mouiller en Norvège (Chamisso, 1813, p. 119), grâce à une paire de bottes de sept lieues.

Ce que les histoires littéraires les plus récentes ont identifié comme les «débuts» de la littérature migratoire, à savoir les textes produits par les travailleurs migrants (ou leurs enfants, dans le cas de Zaimoğlu), est en fait partiellement l'objet d'une (re-)construction s'inscrivant dans une volonté politique d'ouverture et d'actualisation de l'image de la culture de langue allemande. D'une part, les auteurs 
eux-mêmes ont bénéficié d'une forte revalorisation par la création du Prix Chamisso, qui a également favorisé leur visibilité sur le marché du livre, alors que leurs premiers textes avaient mené une existence quasi clandestine, semblable à celle du personnage imaginé par Chamisso. D'autre part, cette littérature qui était d'abord un récit de la migration a été isolée d'autres récits traitant de la même thématique mais signés par des auteurs allemands, et c'est progressivement la question de l'interculturalité et du plurilinguisme qui a justifié son intérêt. Enfin, du point de vue de la périodisation, il convient de distinguer entre la phase de présence effective des travailleurs migrants sur le sol allemand (années 1950-1970) et la production d'une littérature relative à cette époque qui a percé plutôt à partir des années 1990.

\section{Lélargissement vers l'Est: la littérature plurilingue des personnes déplacées}

À partir des années 2000, la littérature de langue allemande connaît une nouvelle phase d'ouverture, cette fois vers l'Est et le centre de l'Europe. Elle pourrait s'expliquer bien sûr par l'élargissement de l'Union européenne et l'adhésion de dix nouveaux États membres en 2004, mais il faut y ajouter un autre aspect, propre à l'histoire migratoire allemande là encore. Entre 1944 et 1950, bien avant la vague migratoire qu'on vient d'examiner dans la première partie, douze millions de personnes issues de communautés minoritaires germanophones arrivent en effet en Allemagne à la suite des mouvements forcés de population en Europe centrale et orientale au sortir de la Seconde Guerre mondiale. Appelés les «expulsés» (Vertriebene), ${ }^{8}$ ils ont été suivis à partir de 1950 par environ cinq millions de Aussiedler, originaires d'Union soviétique, de Roumanie, de Tchécoslovaquie et de Pologne, puis par une dernière vague de moindre envergure, après 1989, de Spätaussiedler. À côté des descendants des travailleurs migrants, les expulsés et leur famille forment le plus important groupe de personnes issues de l'immigration en Allemagne et possèdent pour la plupart la nationalité allemande (cf. Destatis, $1^{\text {er }}$ août 2017). La loi de 1953 (Bundesvertriebenengesetz, BVFG) la leur octroie en effet, les considérant comme membres de la

8. Le terme Vertriebene regroupe des cas fort différents et a fait l'objet de nombreuses contestations, parce qu'il met l'accent sur le statut de victime des expulsés et la responsabilité des pays qu'ils ont dû quitter. Umsiedler lui était d'ailleurs préféré en RDA. Mais Vertriebene a fini par s'imposer, notamment par le biais de la législation (Bundesvertriebenengesetz) de 1953. 
«nation allemande» en raison de leur «ascendance, langue, éducation ou culture». Le niveau de langue requis pour y prétendre est fixé au «niveau seuil» du Cadre européen commun de référence pour les langues, soit B1 (le locuteur sait "poursuivre une interaction» et «faire face» aux situations quotidiennes) (Conseil de l'Europe, 2001, p. 25). Or, la mémoire collective de cette immigration d'aprèsguerre n'a véritablement resurgi dans l'espace public qu'à partir des années 2000, frappée auparavant d'un tabou qui l'avait reléguée principalement dans le cercle familial et associatif (Volkwein, 2012). Alors que leur connaissance de la langue et de la culture allemandes aurait pu jouer en faveur de leur intégration, les expulsés étaient considérés comme traditionnalistes et conservateurs, tenants d'une grande Allemagne à un moment où la République de Bonn voulait marquer sa différence d'avec l'Allemagne nazie.

Dans le champ littéraire, les récits de la «fuite et expulsion» ont longtemps fait défaut, avant de trouver une expression emblématique dans le roman de Günter Grass (1927-2015), Im Krebsgang (2002). Grass n'est pas le seul à s'être emparé du sujet, mais il le fait à un moment décisif où les historiens abordent la question ${ }^{9}$ et avant qu'elle ne prenne une place grandissante par le biais d'expositions et de séries télévisées quelques années plus tard. En 2009, cette littérature a connu une exposition médiatique internationale par la remise du prix Nobel de littérature à Herta Müller (née en 1953 en Roumanie), laquelle a grandi dans un village germanophone du Banat, en Roumanie. Elle évoque dans "Denk nicht dorthin, wo du nicht sollst» comment elle chantait à l'école les chansons de Hoffmann von Fallersleben, les mêmes qu'on apprend à cet âge en Allemagne (Müller, 2013 [2011]). Sa littérature documente non seulement la vie dans une communauté germanophone minoritaire, son expérience de la dictature de Nicolae Ceaușescu et sa fuite en RFA en 1987, mais aussi la création au contact de plusieurs langues ou variétés intralinguistiques. En effet, Müller parlait une variante dialectale de l'allemand à la maison et au village (Banatdeutsch) avant d'apprendre le roumain une fois scolarisée à Timișoara; elle écrit aujourd'hui en allemand standard (Hochdeutsch). Ce plurilinguisme d'écriture lui permet, selon ses propres dires, de «voir le monde avec d'autres yeux» (Müller, 22 février 2011; ma trad.), comme en témoigne très littéralement un passage de son essai «Jedes Wort weiß

9. En particulier Detlef Brandes avec Der Weg zur Vertreibung 1938-1945 publié en 2001. 
etwas vom Teufelskreis». Mise à la porte de son bureau par la Securitate avec laquelle elle refuse de collaborer, elle se retrouve assise sur les marches de son usine à réfléchir à la signification concrète des mots, non seulement en allemand, mais aussi dans la langue de l'artisan qui a construit les «joues» et les «yeux» de l'escalier. Le catalyseur de ses observations devient le mot Treppenwitz, dont on peut noter - ce qui n'est pas dit dans le texte - qu'il s'agit d'un emprunt aux langues romanes, "avoir l'esprit d'escalier». Une autre distinction est venue confirmer en 2013 l'importance nouvelle de cette littérature de langue allemande originaire d'Europe centrale et orientale: le Deutscher Buchpreis décerné à Terézia Mora (née en 1971 en Hongrie) pour Das Ungeheuer, suivi en 2018 du très prestigieux prix Georg Büchner.

Que l'on parle de nos jours d' «Osterweiterung» (Bürger-Koftis, 2009 $)^{10}$, c'est-à-dire d'un élargissement vers l'Est de la littérature allemande à propos de Mora et Müller, qui appartiennent à des communautés germanophones dont la mémoire a été si longtemps occultée, prouve un profond changement de l'identité allemande actuelle et de son rapport au passé. Plus notable encore est l'intégration, dans ce même chapitre de l'histoire littéraire contemporaine, d'autres auteurs arrivés des mêmes pays, avec pourtant une histoire différente. On trouve parmi eux Ilija Trojanow, né en 1965 en Bulgarie, pays qu'il a fui avec ses parents en 1971, d'abord sans vraiment s'arrêter en Allemagne (où ils avaient obtenu l'asile politique), puis y revenant régulièrement, notamment pour ses études. Trojanow est connu pour ses séjours en Afrique et en Inde, dont il a tiré entre autres Der Weltensammler (2006). Dans Die Welt ist groß und Rettung lauert überall (1996), il aborde le dépaysement ressenti à son retour en Bulgarie. Saša Stanišić, né en 1978 en Yougoslavie, a pour sa part fui la guerre civile en 1992 et publié un premier roman très remarqué et récompensé de plusieurs prix, Wie der Soldat das Grammofon reparierte (2006), en partie autobiographique. Arrivée la même année, Lena Gorelik, née en 1981 à Saint-Pétersbourg, s'est fait un nom dans la littérature de voyage ainsi qu'avec Sie können aber gut Deutsch (2012) sur la situation des Allemands allophones en Allemagne. Le point commun de ces auteurs est bien sûr leur usage de la langue allemande comme langue d'écriture, mais aussi une problématisation d'un plurilinguisme d'écriture et d'une hospitalité (non dénuée de tensions) dans la langue allemande.

10. On pourrait penser aussi à l'«Eastern Turn» défini par Brigid Haines (2008). 
La manière dont s'écrit (ou se réécrit) actuellement l'histoire des littératures de langue allemande témoigne d'une relative autonomisation par rapport à l'histoire migratoire qu'on vient d'esquisser, au profit d'une autre périodisation, ${ }^{11}$ servant aussi un agenda politique plus ou moins explicite. Ainsi, le terme "Osterweiterung" (élargissement vers l'Est) a été choisi en écho à l'élargissement de l'Union européenne, comme l'explique Michaela Bürger-Koftis (2009, p. 10), raison pour laquelle les auteurs d'Extrême-Orient et la Japonaise Yoko Tawada en ont été écartés. Louvrage de BürgerKoftis reflète ainsi l'européanisme de la République de Berlin en même temps que le poids de l'Allemagne dans cette nouvelle Europe, puisque le sous-titre, Porträts einer neuen europäischen Generation (Portraits d'une nouvelle génération européenne; ma trad.), sousentend que la nouvelle Europe parle allemand. Mais il arrive aussi que l'histoire littéraire déborde les décisions politiques, en incluant des auteurs issus tant des nouveaux États membres que des pays candidats à l'entrée dans l'Union, comme la Turquie (Zaimoğlu, par exemple). Yasemin Yildiz adopte quant à elle dans Beyond the Mother Tongue une perspective assez différente quoique tout aussi politique. Elle s'intéresse à Zaimoğlu, Özdamar et Tawada, mais justement pas aux auteurs contemporains d'Europe centrale. Elle fonde son paradigme $\mathrm{du}$ "post-monolingue» sur les auteurs praguois du début du XX ${ }^{\mathrm{e}}$ siècle, avec Franz Kafka pour principal représentant du plurilinguisme d'écriture. Ironie du sort, les germanistes français d'après-guerre avaient été accusés d'avoir «fait» de Kafka un auteur allemand, l'égérie de «la grande littérature allemande, celle qui n'avait pas sombré avec le national-socialisme» (Thirouin, 2012, p. 12), passant sous silence l'influence des cultures yiddish et tchèque. L'ouvrage de Yildiz montre comment Kafka est désormais au cœur d'un nouvel infléchissement. La position de Yildiz est certainement la plus radicale quand elle choisit l'allemand comme modèle d'une lingua franca non ethnicisée (2012, p. 19), tandis que les universitaires allemands se contentent pour leur part d'en faire un creuset du plurilinguisme et du transculturalisme. Toutefois, il ne faudrait pas oublier que la littérature est aussi un marché littéraire et une politique culturelle. Dans un dernier point, j'aimerais donc examiner la promotion des

11. Périodisation qui commencerait avec les travailleurs invités dans les années 1980 et 1990 - date non pas de leur arrivée mais de leurs premiers succès - suivis par les auteurs d'Europe centrale et orientale auxquels succèdent aujourd'hui les auteurs qu'on pourrait appeler «de la globalisation», venus librement. 
auteurs allophones qui écrivent en allemand comme ressort d'une nouvelle influence culturelle et linguistique de l'Allemagne.

\section{L'hospitalité dans la langue?}

L'Allemagne se conçoit aujourd'hui comme "terre d'immigration» (Einwanderungsland) mais aussi comme une «société d'immigration" (Einwanderungsgesellschaft). Ces dernières années, la part de personnes d'origine extraeuropéenne a cru de $50 \%$, et bon nombre de personnes résidant en Allemagne ont désormais leurs racines au Moyen-Orient ou en Asie (Destatis, 2017). Bien que l'idée d'une société plurielle ait mis du temps à s'installer dans le discours public, celle-ci transcende désormais les clivages politiques, du moins parmi les grands partis traditionnels (Volksparteien) et leurs alliés. Les gouvernements successifs d'Angela Merkel semblent vouloir tirer des leçons du passé, notamment des problèmes engendrés à l'époque des Gastarbeiter par l'absence de politique d'intégration puis de ce qu'on a appelé le modèle multikulti (modèle de société multiculturelle). Parallèlement au changement de paradigme qu'on vient de montrer pour la littérature, on assiste présentement à la recherche d'un nouveau paradigme national (Leitbild) dans le domaine politique :

Es ist an der Zeit, ein politisches Leitbild zu entwickeln, das plurale Zugehörigkeiten als einen Marker dieses Einwanderungslandes beschreibt. Deutschsein ist heute kulturell, ethnisch, religiös und national vielfältiger geworden. (Bundesregierung, 28 juin 2016)

[il est temps de développer un nouveau paradigme politique, dans lequel la pluralité des appartenances serait le propre de notre société d'immigration. Être allemand signifie aujourd'hui des choses très variées sur le plan culturel, ethnique, religieux et national.] (ma trad.)

La réforme du code de la nationalité sous le gouvernement de Gerhard Schröder (1998-2005) a déjà modifié par la loi ce qu' «être allemand» veut dire. Alors que le droit du sang (jus sanguinis) a longtemps prévalu en Allemagne, le droit du sol (jus soli) est également pris en considération depuis 2000. Létape suivante est de fonder la communauté nationale sur la langue, laquelle serait un ferment de solidarité entre populations d'origines différentes, possédant ou non la nationalité allemande - parfois d'ailleurs au détriment d'une lutte pour les mêmes droits civiques (ibid.). Or, pour s'imposer, un tel paradigme a besoin qu'on l'ancre dans l'histoire et la culture nationale, d'autant plus que l'idée d'une «identité allemande» n'est pas abandonnée: on voudrait faire du pluralisme le typiquement 
allemand, soit un oxymore nécessitant une petite gymnastique de l'esprit, ou plutôt une politique linguistique et culturelle d'envergure.

Pour justifier l'ouverture des frontières en 2015 et l'accueil de plus d'un million de réfugiés, la chancelière Angela Merkel a pu s'appuyer sur l'histoire de son pays. Pas seulement l'histoire migratoire, dont on vient d'évoquer les grandes lignes, mais aussi la réunification, thème traditionnel de l'allocution du Nouvel An qui, depuis 2014, se trouve systématiquement liée au thème de l'intégration. C'est le cas dans l'extrait suivant, où Angela Merkel évoque le sort d'un citoyen allemand d'origine kurde, qu'elle met en parallèle avec les revendications des citoyens de RDA avant la chute du Mur :

Vor vielen Jahren sei er aus dem Irak geflohen - unter sehr schwierigen Bedingungen. Unter Lebensgefahr. Er habe gesagt, das Wichtigste sei für ihn in Deutschland, dass seine Kinder hier ohne Furcht aufwachsen könnten. Das ist vielleicht das größte Kompliment, das man unserem Land machen kann: dass die Kinder Verfolgter hier ohne Furcht groß werden können. Und das war auch ein Motiv der vielen Menschen, die vor 25 Jahren in der DDR jeden Montag auf die Straße gingen. (Bundesregierung, 31 décembre 2014)

[Il y a de cela des années, cet homme a fui l'Irak, dans des conditions très difficiles. Au péril de sa vie. Il a affirmé que le plus important pour lui, c'était qu'en Allemagne ses enfants puissent grandir sans crainte. Voilà le plus beau compliment qu'on puisse faire à notre pays : les enfants de personnes persécutées peuvent y grandir en paix. C'était aussi l'une des motivations des nombreuses personnes qui, il y a vingt-cinq ans en RDA, descendaient tous les lundis dans la rue.] (ma trad.)

Alors que pendant la décennie 1990 les efforts financiers et économiques exigés par la réunification avaient fait de l'Allemagne "l'homme malade de l'Europe", quelque trente ans plus tard, l'Allemagne aime se prévaloir de son savoir-faire en matière d'intégration, de la même façon que l'Office franco-allemand pour la Jeunesse propose désormais ses services dans les pays de l'ex-Yougoslavie, sur la base de son expérience dans la gestion de conflits. ${ }^{12}$

Certains auteurs allophones ayant opté pour la langue allemande comme langue de création sans y avoir été poussés par l'exil ou la migration contrainte se sont expliqués sur leur choix. Je prendrai ici

12. Cf. Office franco-allemand pour la jeunesse (2017), où il est dit notamment que «ces programmes permettent de proposer la relation franco-allemande comme source d'inspiration aux citoyens de cette région [les Balkans]». 
deux exemples, Sharon Dodua Otoo et Yoko Tawada, dont les arguments sont au cœur de l'actuelle promotion de la littérature de la migration visant à inciter de nouveaux auteurs à écrire en allemand et ainsi promouvoir l'allemand comme nouvelle langue globale. Arrivées en Allemagne dans le cadre d'une migration individuelle librement choisie, ces deux auteures bénéficient d'une audience privilégiée, illustrant pour l'une la promotion par le marché littéraire, à la faveur d'un prix littéraire de renom (prix Ingeborg Bachmann de 2016) et pour l'autre par le milieu universitaire, son œuvre relativement peu connue du grand public ayant réussi à se faire une place de choix dans les recherches récentes ${ }^{13}$. Sharon Dodua Otoo, qui a d'abord publié dans sa première langue, l'anglais, s'est tournée vers l'allemand après s'être installée à Berlin. On peut d'ailleurs rappeler au passage l'importance symbolique du choix de Berlin comme capitale de l'Allemagne réunifiée, non pas en raison de son poids historique, mais parce qu'elle était à reconstruire. Elle incarnait ainsi le projet politique et sociétal en cours, tout en devenant très attractive pour des projets étrangers. La ville, parfois classée parmi les «métropoles mondiales ", est sans conteste la capitale de cette nouvelle Allemagne transculturelle. Sharon Dodua Otoo a expliqué lors du festival Mixed Zone, le 26 octobre $2017^{14}$, que c'est une certaine «qualité des débats » qui l'a attirée à Berlin, qualité non seulement des débats publics ou politiques, mais des échanges plus informels. Plutôt que de glisser rapidement vers un consensus, les discussions supporteraient, selon elle, le débat contradictoire et l'interrogation critique du passé. Même s'il faut se garder d'accorder une importance disproportionnée à une impression peut-être fugace et fondée sur l'expérience individuelle, l'argument de Dodua Otoo n'est en fait pas si personnel. Elle l'ancrait d'ailleurs dans le récent débat autour du Forum Humboldt installé dans le Château de Berlin et qui doit accueillir à partir de 2019 des expositions sur les cultures extraeuropéennes. Ce projet a été vivement critiqué, entre autres par l'historienne de l'art et professeure au Collège de France Bénédicte Savoy, pour l'amnésie dont il fait preuve quant à la provenance des œuvres et à la manière dont elles

13. Un premier colloque lui a été consacré sous la direction de Douglas Slaymaker (Slaymaker, 2007), suivi par d'autres, notamment en France (2009) et en Autriche (2011). En Allemagne, elle a bénéficié d'une résidence à l'Université de Hambourg (2011) et a tenu une série de conférences poétologiques à l'Université de Tübingen (Tawada, 1998).

14. Entretien avec Laura Beck à la Cité Miroir de Liège, en Belgique. 
ont été acquises pendant la période coloniale. Selon Dodua Otoo, le débat sur le passé colonial de l'Allemagne, et plus généralement l'examen critique de l'histoire nationale, serait sans comparaison avec ce qu'elle a pu connaître au Royaume-Uni. L'Allemagne se distingue certainement, y compris des autres pays de langue allemande, par son rapport critique au récit national, hérité de la dénazification et des programmes de «ré-éducation» menée par les Alliés. Les références aujourd'hui incontournables sur le travail de mémoire et la mémoire collective sont l'œuvre d'historiens ainsi que d'historiens de la culture allemands (on peut penser notamment à Aleida Assmann et Jan Assmann). Dans nombre de pays, l'interrogation critique des pages sombres de l'histoire court le risque de se voir accusée d'antipatriotisme, tandis qu' «être allemand» a longtemps signifié accepter une part de culpabilité pour les crimes nazis. Le rapport problématique au passé a contribué aussi à faire de l'avenir un projet ouvert, nécessairement en rupture avec l'histoire, du moins l'histoire récente. Jusqu'à aujourd'hui, la constitution allemande reste une «Loi fondamentale» (Grundgesetz) dont le dernier article affirme le caractère provisoire : «la présente Loi fondamentale [...] devient caduque le jour de l'entrée en vigueur d'une constitution adoptée par le peuple allemand en pleine liberté de décision» (Bundestag, 2012; ma trad.). L'adhésion aux valeurs démocratiques énoncées dans cette Loi fondamentale, appelée Verfassungspatriotismus par les politologues et philosophes Jürgen Habermas, Dolf Sternberger et Jürgen Seifert, a tenu lieu de sentiment patriotique pendant des décennies pour nombre d'Allemands. Elle a facilité l'introduction du jus soli et l'accueil d'étrangers. On peut donc penser, à partir des déclarations de Dodua Otoo, que le caractère longtemps inachevé de l'Allemagne d'après-guerre, une certaine politique du projet indissociablement liée à une révision critique du passé, est devenu un terreau propice à l'accueil de nouvelles populations en quête d'un changement politique dans leur propre pays.

Un autre argument est formulé par la Japonaise Yoko Tawada (née en 1960), arrivée en Allemagne au début des années 1980. Tawada avait le libre choix de son lieu de résidence et affirme avoir préféré l'Allemagne à la Russie (dont elle connaissait pourtant la langue et la littérature par ses études) ou à la France (où elle a néanmoins séjourné). Ses œuvres, pour beaucoup essayistes ou poétiques, ne manifestent toutefois pas vraiment un choix arrêté en faveur de la langue allemande, qui devient plutôt le lieu d'une réflexion sur 
les rapports entre idéogrammes et alphabet latin et un terrain de jeu où l'on glisse d'un système de signes à un autre (Tawada, 2002, 2010, 2016). Son argument subjectif et personnel rejoint une idée répandue jusque chez les historiens de la littérature de langue allemande. La thèse de Heinz Schlaffer résumée par le titre (traduit) de son ouvrage La courte histoire de la littérature allemande postule la faible amplitude chronologique de la littérature de langue allemande à travers la comparaison avec d'autres littératures nationales - en particulier française et anglaise. Pour Schlaffer, les débuts médiévaux ne sont qu'une reconstitution a posteriori des hommes de lettres du $\mathrm{XIX}^{\mathrm{e}}$ siècle, dans le désir de forger une littérature nationale et de la doter de racines anciennes (2003 [2002], p. 22-23). Son évolution ultérieure n'est aucunement de qualité constante, comme un esprit nationaliste aimerait le croire, mais faite d'apogées et de périodes de déclin, voire d'insignifiance. Et d'apogées, Schlaffer n'en admet que deux : le XVIII ${ }^{\mathrm{e}}$ siècle et le début du XX $\mathrm{XX}^{\mathrm{e}}$, qu'il caractérise comme des moments d'ouverture aux influences étrangères, aux Lumières françaises d'une part (ibid., p. 110), aux écrivains juifs d'Europe centrale (Vienne et Prague) d'autre part (ibid., p. 134). Schlaffer finit donc par pousser à son comble sa thèse initiale : cette littérature n'est pas seulement courte, elle est inexistante si on veut lui donner le nom de «littérature allemande» et ainsi refuser de voir qu'elle n'a pu se construire qu'au contact d'influences étrangères. La périodisation proposée par Schlaffer, de même que ses préférences personnelles ${ }^{15}$, sont éminemment discutables et cherchent à l'être, son ouvrage flirtant clairement avec le genre du pamphlet. Mais une idée convainc certainement et peut être reliée au propos de Tawada : parler de «littérature allemande» est problématique, parce qu'une codification précoce et collective, comme cela a pu se faire en France, lui a manqué :

Les écrivains allemands ressemblent à leurs personnages, en cela qu'ils ne peuvent recourir à une langue littéraire évoluée comme elle avait par exemple cours en France depuis le XVII e siècle; ils sont bien plutôt contraints d'inventer une nouvelle langue. ${ }^{16}$ (ibid., p. 92)

15. Par exemple, la seconde moitié du $\mathrm{XX}^{\mathrm{e}}$ siècle n'aurait selon lui connu aucun bon écrivain.

16. «Deutsche Dichter ähneln ihren Figuren darin, dass sie sich auf keine entwickelte literarische Sprache verlassen können, wie sie etwa in Frankreich seit dem 17. Jahrhundert geläufig ist; vielmehr müssen sie immer aufs neue eine neue Sprache erfinden». 
Il n'existe ainsi pas de variété de la langue allemande qui servirait d'étalon aux autres, même si l'Allemagne joue certainement un rôle dominant. Et il n'y a pas de comparaison possible avec le «français de France» et son influence sur la francophonie, tantôt modèle, tantôt repoussoir, par exemple pour les écrivains québécois qui ont pu considérer la langue française littéraire comme «trop polie, trop cultivée, trop usée, trop étiolée, trop instruite, trop codifiée, trop propriété privée, trop correcte pour l'usage qu['ils veulent] en faire» (Jacques Godbout cité par Gauvin, 2000, p. 38). L'importance des parlers régionaux dans l'aire germanophone - qu'il s'agisse de particularités lexicales ou phonétiques ou de dialectes à part entière peut laisser croire à Tawada que la langue et ses locuteurs sont plus flexibles à l'endroit des germanophones non natifs, ceux-ci risquant toutefois de se voir opposer l'absence d'ancrage local, de terroir de leur langue, comme c'est le cas dans le roman de Terézia Mora, Alle Tage $^{17}$. Si on suit Schlaffer, la littérature de langue allemande ne doit ses âges d'or successifs qu'à des influences mêlées et des auteurs venus d'ailleurs, une thèse prolongée par Tina Hartmann, qui identifie la littérature contemporaine de la migration avec un troisième âge d'or (2016).

\section{Conclusion}

Alors que diverses tendances traversent la littérature contemporaine (comme la ruralité, les nouvelles technologies, la critique du monde du travail; cf. Caduff et Vedder, 2017), le plurilinguisme et le transculturel connaissent pour leur part un intérêt sans égal en Allemagne. J'ai pu montrer dans ce qui précède qu'on assiste à la réécriture de l'histoire des migrations et de l'histoire littéraire pour accueillir les œuvres de plus en plus nombreuses d'auteurs allophones et d'autres germanophones extérieurs aux pays de langue allemande. Si les débuts de la dite Gastarbeiterliteratur ont été difficiles, car souvent empêchés à un moment où la langue allemande est «réservée » aux locuteurs natifs, l'ouverture vers l'Est (Osterweiterung) s'est faite bien plus facilement, préparant le terrain à une autre vague, celle des auteurs allophones de la globalisation qui choisissent l'allemand

17. Le personnage de traducteur, Abel Nema, héros du roman de Mora, vit au croisement de dix langues apprises uniquement en laboratoire de langue et qu'il parle sans ancrage géographique ni accent, «comme quelqu'un qui ne viendrait de nulle part» (Mora, 2004, p. 13; ma trad.). On pourrait penser aussi au personnage d'Irene dans Reisende auf einem Bein de Herta Müller (1989). 
comme langue d'accueil. La diffusion de la langue allemande dans le monde n'est que rarement thématisée et, pendant longtemps, elle n'a pas fait l'objet d'études approfondies comparables à celles dont on dispose pour l'anglais, le français ou l'espagnol (Ammon, 2003, p. 345). Le nombre de locuteurs natifs ayant l'allemand comme langue première est estimé à une centaine de millions (ibid., p. 346), ce qui fait de l'allemand une des douze langues les plus parlées au monde. Mais c'est évidemment le nombre de locuteurs seconds, particulièrement hors des frontières nationales, qui compte le plus pour mesurer l'importance d'une langue. L'allemand bénéficie pour partie de la puissance économique des pays de l'aire germanophone, au point d'être dans certains secteurs un atout à l'embauche. Il compte parmi les langues enseignées à l'école secondaire et dans le supérieur partout où ces structures existent, mais son importance comme langue scientifique est en net recul par rapport à ce qu'elle était au début du $\mathrm{XX}^{\mathrm{e}}$ siècle. Au sein de l'ONU, il ne compte pas parmi les six langues officielles.

$\mathrm{Si}$ on change d'échelle pour observer ce qu'il en est en Europe, l'allemand prend alors une tout autre envergure, dépassant numériquement et économiquement l'anglais (une situation qui pourrait s'accentuer avec la sortie du Royaume-Uni de l'Union européenne). La politique culturelle et linguistique des pays de langue allemande a pourtant une ambition plus vaste. Sa dernière inflexion majeure remonte au gouvernement Schröder qui, sous le nom de Konzeption 2000, a modernisé le pilier culturel de la politique extérieure de l'Allemagne en mettant l'accent non plus sur la diffusion de la culture nationale, mais sur les échanges culturels et la coopération (Bauer, 2003, p. 138). Ceci passe d'une part par la promotion de la culture en provenance de l'Allemagne comme aspect de la culture européenne et, d'autre part, par la conviction qu'il faut apprendre à apprendre des cultures extraeuropéennes (ibid.). Des organismes publics (principalement l'Institut Goethe et le DAAD - Deutscher Akademischer Austauschdienst) travaillent conjointement avec des fondations privées (Robert Bosch-Stiftung) à la promotion d'«études germaniques interculturelles» (interkulturelle Germanistik) à travers le monde, qui contribuent à changer l'image parfois dépréciée ou poussiéreuse de la culture de langue allemande.

C'est dans ce contexte qu'il faut examiner la place de la littérature de la migration et sa forte promotion sur le marché du livre. Celle-ci englobe à la fois la médiatisation de ses auteurs (par le biais 
de prix littéraires), un soutien financier (par des programmes publics et des fondations privées), une théorisation par le milieu universitaire et un infléchissement du discours politique. Croisant les ambitions d'un renouvellement de son image (pour soi et vers l'extérieur), la redéfinition actuelle de l'identité allemande par la littérature cherche à faire de l'allemand "le symbole de la pluralité des cultures", un peu à la façon dont le chercheur québécois Nicolas Van Schendel a pu le revendiquer pour le français (Gauvin, 2000, p. 45). Plusieurs projets politiques et acteurs culturels se disputent cette ambition et lui donnent un sens très différent, de l'utopie d'une germanitude désethnicisée à l'incarnation du transculturel dans l'«être allemand». Espérant offrir aux auteurs d'un monde globalisé une alternative à un marché anglophone saturé, l'Allemagne semble certes prête à abandonner l'idée de l'appartenance par l'origine, mais seulement au profit d'un accroissement de l'influence culturelle et linguistique qui lui a longtemps manqué. Une façon bien paradoxale d'accepter de s'y perdre pour s'y retrouver ${ }^{18}$.

\section{Références}

Ammon, Ulrich (2003). «Die Stellung der deutschen Sprache in der Welt» [La place de la langue allemande dans le monde]. In A. Wierlacher et A. Bogner, dir. Handbuch interkulturelle Germanistik. Stuttgart, J.B. Metzler, p. 345-354.

Bauer, Gerd Ulrich (2003). «Auswärtige Kulturpolitik» [Politique culturelle étrangère]. In A. Wierlacher et A. Bogner, dir. Handbuch interkulturelle Germanistik. Stuttgart, J.B. Metzler, p. 132-143.

Brandes, Detlef (2001). Der Weg zur Vertreibung 1938-1945 [Le chemin vers l'expulsion 1938-1945]. Munich, Oldenbourg.

Bundesministerium für Justiz und Verbraucherschutz (s.d.). «Gesetz über die Angelegenheiten der Vertriebenen und Flüchtlinge (Bundesvertriebenengesetz - BVFG) § 6 Volkszugehörigkeit» [Loi concernant les personnes expulsées]. Disponible à <https://www.gesetzeim-internet.de/bvfg/_6.html> [consulté le 13 janv. 2018].

Bundesregierung (31 décembre 2014). «Neujahrsansprache 2015» [Discours du Nouvel An 2015]. Disponible à <https://www.bundesregierung.de/ breg-de/service/bulletin/neujahrsansprache-2015-798300> [consulté le 6 avril 2019].

Bundesregierung (28 juin 2016). «Was ist deutsch im Jahr 2016 - die

18. Je fais ici écho au thème du colloque international «La traduction transculturelle et interlinguistique : s'y perdre et s'y retrouver» qui s'est tenu à l'Université de Moncton en novembre 2017. Le présent article découle de l'appel à articles diffusé à la suite de ce colloque. 
Vermessung der Einwanderungsgesellschaft» [Qu'est-ce qui est allemand en 2016 - Portrait d'une société d'immigration]. Disponible à <https://www.bundesregierung.de/Content/DE/Artikel/IB/Artikel/ Allgemein/2016-06-28-pm-das-neue-deutsche-wir.html> [consulté le 13 janv. 2018].

Bundestag (2012). Loi fondamentale pour la République fédérale d'Allemagne. Trad. C. Autexier, M. Fromont, C. Grewe et O. Jouanjan. Disponible à <https://www.bundestag.de/blob/189762/ f0568757877611b2e434039d29a1a822/loi_fondamentale-data.pdf> [consulté le 13 janv. 2018].

Bürger-Koftis, Michaela, dir. (2009). Eine Sprache - viele Horizonte. Die Osterweiterung der deutschsprachigen Literatur. Porträts einer neuen europäischen Generation [Une langue - des horizons multiples. Lélargissement vers l'Est de la littérature de langue allemande. Portraits d'une nouvelle génération européenne]. Vienne, Praesens Verlag.

Caduff, Corinna et Ulrike Vedder, dir. (2017). Gegenwart schreiben. Zur deutschsprachigen Literatur 2000-2015 [Écrire le contemporain. La littérature allemande 2000-2015]. Paderborn, Wilhelm Fink.

Chamisso, Adelbert von (2003 [1813]). Peter Schlemibls wundersame Geschichte [L'étrange histoire de Peter Schlemihl]. Francfort-sur-leMain, Suhrkamp.

Conseil de l'Europe (2001). Cadre européen commun de référence pour les langues. Disponible à <https://rm.coe.int/16802fc3a8> [consulté le 6 avril 2019].

Dodua Otoo, Sharon. «Herr Gröttrup setzt sich hin»[Monsieur Gröttrup s'assoit]. Disponible à <http://files2.orf.at/vietnam2/files/ bachmannpreis/201619/herr_grttrup_setzt_sich_hin_sharon_dodua_ otoo_439620.pdf> [consulté le 6 avril 2019].

Gauvin, Lise (2000). Langagement. L'écrivain et la langue au Québec. Montréal, Les éditions du Boréal.

Gorelik, Lena (2012). Sie können aber gut Deutsch [Comme vous parlez bien allemand]. Munich, Pantheon Verlag.

Grass, Günter (2002). Im Krebsgang [En crabe]. Göttingen, Steidl.

Haines, Brigid (2008). «The Eastern Turn in Contemporary German, Swiss and Austrian Literature». Debatte. Journal of Contemporary Central and Eastern Europe, 16, 2, p. 135-149.

Hartmann, Tina (2016). Deutsch als Literaturheimat. Transkulturelle Literatur interkultureller Autoren [L'allemand comme patrie littéraire. Littérature transculturelle d'auteurs interculturels]. Contribution au Congrès mondial de l'association de littérature comparée (ICLA 2016). Inédit.

Herrmann, Leonhard et Silke Horstkotte (2016). Gegenwartsliteratur. Eine Einfübrung [La littérature contemporaine. Une introduction]. Stuttgart, J.B. Metzler. 
Kaube, Jürgen (2016). «Manchmal wache ich auf und denke : Heute bin ich ein $\mathrm{Ei}$ » [Parfois je me réveille en pensant : aujourd'hui je suis un oeuf]. Frankfurter Allgemeine Zeitung, 5 juillet. Disponible à <https://www.faz. net/aktuell/feuilleton/buecher/autoren/die-bachmann-preistraegerinsharon-dodua-otoo-14323628.html> [consulté le 5 juillet 2019].

Keiner, Sabine (1999). «Von der Gastarbeiterliteratur zur Migrantenund Migrationsliteratur. Literaturwissenschaftliche Kategorien in der Krise?» [De la littérature des travailleurs invités à la littérature de migrants et de la migration. Catégories de l'analyse littéraire en crise?]. Sprache und Literatur, 30, 1, p. 13-14.

Kilchmann, Esther (2017). "Von der Erfahrung zum Experiment: literarische Mehrsprachigkeit 2000-2015» [De l'expérience à l'expérimentation : le plurilinguisme littéraire 2000-2015]. In C. Caduff et U. Vedder, dir. Gegenwart schreiben. Zur deutschsprachigen Literatur 20002015. Paderborn, Wilhelm Fink, p. 177-186.

Mora, Terézia (2006 [2004]). Alle Tage [Tous les jours]. Munich, btb Verlag. Mora, Terézia (2013). Das Ungeheuer [Le monstre], Munich, Luchterhand Literaturverlag.

Müller, Herta (2013 [2009]). «Jedes Wort weiß etwas vom Teufelkreis» [Chaque mot en sait un peu sur le cercle vicieux]. In $\mathrm{H}$. Müller, Immer derselbe Schnee und immer derselbe Onkel. Francfort-sur-le-Main, Fischer, p. 7-21.

Müller, Herta (22 février 2011). "Sprache ist Heimat» [La langue est une patrie]. Disponible à <https://www.youtube.com/watch?v $=y t T 31 z 1 q W r c>$ [consulté le 13 janv. 2018].

Müller, Herta (2013 [2011]). «Denk nicht dorthin, wo du nicht sollst. Dankrede zur Verleihung des Hoffmann-von-Fallersleben-Preises für zeitkritische Literatur» [ $\mathrm{Ne}$ mène pas tes pensées là où tu n'es pas censé les mener. Discours de remerciement pour le prix Hoffmann-vonFallersleben pour la littérature critique]. In $\mathrm{H}$. Müller, Immer derselbe Schnee und immer derselbe Onkel. Francfort-sur-le-Main, Fischer, p. 2541.

Noack, Hans-Georg (1973). Benvenuto heißt willkommen [Benvenuto c'est bienvenue]. Baden-Baden, Signal.

Office franco-allemand pour la jeunesse (11 septembre 2017). «Création d'une structure favorisant les échanges de jeunes dans les Balkans: les Premiers ministres serbe et albanais rencontrent les Secrétaires généraux de l'OFAJ». Disponible à <https://www.ofaj.org/ressources/ creation-d-une-structure-favorisant-les-echanges-de-jeunes-dansles-balkans-les-premiers-ministres-serbe-et-albanais-rencontrent-lessecretaires-generaux-de-1-ofaj.html> [consulté le 6 avril 2019].

Oltmer, Jochen (2012). «Einführung: Migrationsverhältnisse und Migrationsregime nach dem Zweiten Weltkrieg» [Introduction: Rapports de migrations et régimes de migration après la Seconde 
Guerre mondiale]. In J. Oltmer, A. Kreienbrink et C. Sanz Díaz, dir. Das "Gastarbeiter"-System. Arbeitsmigration und ibre Folgen in der Bundesrepublik Deutscbland und Westeuropa. Munich, Oldenburg Wissenschaftsverlag GmbH, p. 9-24.

Özdamar, Emine Sevgi (1992). Das Leben ist ein Karawanserei [La vie est un caravansérail]. Cologne, Kiepenheuer und Witsch.

Özdamar, Emine Sevgi (1998). Die Brücke vom goldenen Horn [Le pont de la corne d'or]. Cologne, Kiepenheuer und Witsch.

Reimann, Daniela (2017). Die Literaturvermittlung in der auswärtigen Kulturpolitik Deutschlands. Eine Betrachtung ibrer Entwicklung, Instrumente und Tendenzen [La médiation littéraire dans la politique culturelle étrangère allemande. Une analyse de son évolution, de ses instruments et de ses tendances]. Würzburg, Königshausen \& Neumann.

Schlaffer, Heinz (2003 [2002]). Die kurze Geschichte der deutschen Literatur [La courte histoire de la littérature allemande]. Munich, dtv.

Slaymaker, Douglas (2007). Yoko Tawada. Voices from Everywhere. Lanham, Lexington Books.

Stanišić, Saša (2006). Wie der Soldat das Grammofon reparierte [Le soldat et le gramophone]. Munich, Luchterhand Literaturverlag.

Statistisches Bundesamt - Destatis (17 décembre 2013). Migration hat eine lange Tradition in Deutschland [La migration a une longue tradition en Allemagne]. Disponible à <https://www.destatis.de/DE/PresseService/ Presse/Pressemitteilungen/2013/12/PD13_430_122.html> [consulté le 13 janv. 2018].

Statistisches Bundesamt - Destatis (1 ${ }^{\text {er }}$ août 2017). Bevölkerung mit Migrationshintergrund um $8,5 \%$ gestiegen [La population avec un arrièreplan migratoire augmente de $8,5 \%$ ]. Disponible à <https://www. destatis.de/DE/PresseService/Presse/Pressemitteilungen/2017/08/ PD17_261_12511.html> [consulté le 13 janv. 2018].

Tantow, Lutz (1984). «In den Hinterhöfen der deutschen Sprache. Ein Streifzug durch die deutsche Literatur von Ausländern » [Dans l'arrièrecour de la langue allemande. Un aperçu de la littérature allemande écrite par des étrangers]. Die Zeit, 6 avril.

Tawada, Yoko (1998). Verwandlungen. Tübinger Poetikvorlesungen [Transformations. Conférences poétologiques de Tübingen]. Tübingen, Konkursbuch Verlag Claudia Gehrke.

Tawada, Yoko (2002). Überseezungen [Traduction - langues ultramarines]. Tübingen, Konkursbuch Verlag Claudia Gehrke.

Tawada, Yoko (2010). Abenteuer der deutschen Grammatik [Aventure(s) de la grammaire allemande]. Tübingen, Konkursbuch Verlag Claudia Gehrke.

Tawada, Yoko (2016). akzentfrei [sans accent]. Tübingen, Konkursbuch Verlag Claudia Gehrke.

Thirouin, Marie-Odile (2012). «Introduction». In H. Binder, dir. Kafka 
parlait-il tchèque? La socialisation des écrivains de langue allemande à Prague. Trad. M.-O. Thirouin, Paris, Classiques Garnier, p. 7-18.

Trojanow, Ilija (1996). Die Welt ist groß und Rettung lauert überall [Le monde est grand et le salut partout]. Munich, Carl Hanser.

Trojanow, Ilija (2006). Der Weltensammler [Le collectionneur de mondes]. Munich, Carl Hanser.

Volkwein, Alice (2012). (Dis)cours mémoriel de la fuite et expulsion dans l'Allemagne unifiée (1989-2005). Thèse de doctorat, Département d'études germaniques, Université Sorbonne Nouvelle. Inédit.

Wehler, Hans-Ulrich (2008). Bundesrepublik und DDR. 1949-1990 [République fédérale allemande et RDA. 1949-1990]. Munich. C.H. Beck.

Wierlacher, Alois und Andrea Bogner, dir. (2003). Handbuch interkulturelle Germanistik [Les études germaniques interculturelles]. Stuttgart, J.B. Metzler.

Wikipédia (s. d.). «Rafik Schami». Disponible à <https://de.wikipedia.org/ wiki/Rafik_Schami> [consulté le 14 janv. 2018].

Yildiz, Yasemin (2012). Beyond the Mother Tongue. The Postmonolingual Condition, New York, Fordham University Press.

Zaimoğlu, Feridun (2013 [1995]). Kanak Sprak. 24 Mißtöne vom Rande der Gesellschaft [Langue kanak. 24 fausses notes des marges de la société]. Berlin, Rotbuch Verlag.

\section{Sarah Neelsen}

Département d'études germaniques Université Sorbonne Nouvelle - Paris 3 Paris, France sarah.neelsen@sorbonne-nouvelle.fr 